\title{
Existence of Positive Solutions for a Kind of Fractional Boundary Value Problems
}

\author{
Hongjie Liu, ${ }^{1}$ Xiao Fu, ${ }^{2}$ and Liangping $\mathbf{Q i}^{2}$ \\ ${ }^{1}$ School of Land Science and Technology, China University of Geosciences, Beijing 10083, China \\ ${ }^{2}$ School of Science, China University of Geosciences, Beijing 10083, China \\ Correspondence should be addressed to Hongjie Liu; 1_hongjie@163.com
}

Received 18 March 2014; Accepted 26 April 2014; Published 20 May 2014

Academic Editor: Yonghui Xia

Copyright (c) 2014 Hongjie Liu et al. This is an open access article distributed under the Creative Commons Attribution License, which permits unrestricted use, distribution, and reproduction in any medium, provided the original work is properly cited.

\begin{abstract}
We are concerned with the following nonlinear three-point fractional boundary value problem: $D_{0+}^{\alpha} u(t)+\lambda a(t) f(t, u(t))=0$, $0<t<1, u(0)=0$, and $u(1)=\beta u(\eta)$, where $1<\alpha \leq 2,0<\beta<1,0<\eta<1, D_{0+}^{\alpha}$ is the standard Riemann-Liouville fractional derivative, $a(t)>0$ is continuous for $0 \leq t \leq 1$, and $f \geq 0$ is continuous on $[0,1] \times[0, \infty)$. By using Krasnoesel'skii's fixed-point theorem and the corresponding Green function, we obtain some results for the existence of positive solutions. At the end of this paper, we give an example to illustrate our main results.
\end{abstract}

\section{Introduction}

Fractional differential equations have been of great interest recently. With the development of nonlinear science, the researchers found that nonlinear fractional differential equations could describe something's changing rules more accurately. Therefore, it is significant to study nonlinear fractional differential equations to solve the nonlinear problems. Recently, many researchers paid attention to existence and multiplicity of solution of the boundary value problem for fractional differential equations with different boundary conditions, such as [1-10]. Bai and Lü [1] investigated the existence and multiplicity of positive solutions for nonlinear fractional boundary value problem:

$$
\begin{gathered}
D_{0+}^{\alpha} u(t)+f(t, u(t))=0, \quad 0<t<1, \\
u(0)=u(1)=0,
\end{gathered}
$$

where $1<\alpha \leq 2$ is a real number, $D_{0+}^{\alpha}$ is the standard Riemann-Liouville fractional derivative, and $f:[0,1] \times$ $[0, \infty) \rightarrow[0, \infty)$ is continuous. By means of some fixedpoint theorems on cone, some existence and multiplicity results of positive solutions are obtained. Agarwal et al. [2] investigated the existence of positive solutions for the singular fractional boundary value problem:

$$
\begin{gathered}
D_{0+}^{\alpha} u(t)+f\left(t, u(t), D^{\mu} u(t)\right)=0, \quad 0<t<1, \\
u(0)=u(1)=0,
\end{gathered}
$$

where $1<\alpha \leq 2, \mu>0$ are real numbers, $f$ is positive, and $D_{0+}^{\alpha}$ is the standard Riemann-Liouville fractional derivative. By means of a fixed point theorem on cone, the existence of positive solutions is obtained. Delbosco and Rodino [3] investigated the nonlinear Dirichlet-type problem:

$$
\begin{gathered}
x^{\alpha-1}\left(D_{0+}^{\alpha} y\right)(x)=f(y(x)), \quad 1<\alpha<2,0 \leq x \leq 1, \\
y(0)=y(1)=0 .
\end{gathered}
$$

They proved that if $f(y)$ is a Lipschitzian function, then the problem has at least one solution $y(x)$ in a certain subspace of $C[0,1]$. In this paper, we study the following three-point fractional boundary value problem. Consider

$$
\begin{gathered}
D_{0+}^{\alpha} u(t)+\lambda a(t) f(t, u(t))=0, \quad 0<t<1, \\
u(0)=0, \quad \beta u(\eta)=u(1),
\end{gathered}
$$


where $1<\alpha \leq 2,0<\beta<1,0<\eta<1, D_{0+}^{\alpha}$ is the standard Riemann-Liouville fractional derivative, $a(t)>0$ is continuous, and $f \geq 0$ is continuous on $[0,1] \times[0, \infty)$. By using Krasnoesel'skii's fixed-point theorem, we get the existence of at least one positive solution.

\section{Background Materials and Preliminaries}

For convenience of the readers, we present here the necessary definitions from fractional calculus theory. These definitions can be found in the recent literature [1-5].

Definition 1. The fractional integral of order $\alpha>0$ of a function $y:(0, \infty) \rightarrow R$ is given by

$$
I_{0+}^{\alpha} y(t)=\frac{1}{\Gamma(\alpha)} \int_{0}^{t}(t-s)^{\alpha-1} y(s) d s,
$$

provided that the right side is pointwise defined on $(0, \infty)$.

Definition 2. The fractional derivative of order $\alpha>0$ of a function $y:(0, \infty) \rightarrow R$ is given by

$$
D_{0+}^{\alpha} y(t)=\frac{1}{\Gamma(n-\alpha)}\left(\frac{d}{d t}\right)^{n} \int_{0}^{t} \frac{y(s)}{(t-s)^{\alpha-n+1}} d s, \quad n=[\alpha]+1,
$$

where $n=[\alpha]+1$, provided that the right side is pointwise defined on $(0, \infty)$.

Lemma 3. Let $\alpha>0$, if we assume $u \in C(0,1) \cap L(0,1)$, then the fractional differential equation $D_{0+}^{\alpha} u(t)=0$ has

$$
\begin{array}{r}
u(t)=C_{1} t^{\alpha-1}+C_{2} t^{\alpha-2}+\cdots C_{N} t^{\alpha-N}, \\
C_{i} \in R, \quad i=1,2, \ldots, N
\end{array}
$$

as unique solutions.

Lemma 4. Assume that $u \in C(0,1) \cap L(0,1)$ with a fractional derivative of order $\alpha>0$ that belongs to $C(0,1) \cap L(0,1)$. Then

$$
I_{0+}^{\alpha} D_{0+}^{\alpha} u(t)=u(t)+C_{1} t^{\alpha-1}+C_{2} t^{\alpha-2}+\cdots C_{N} t^{\alpha-N}
$$

for some $C_{i} \in R, i=1,2, \ldots, N$.

Definition 5. Let $E$ be a real Banach space. A nonempty closed convex set $K \subset E$ is called cone of $E$ if it satisfies the following conditions:

(1) $x \in K, \sigma>0$ implies $\sigma x \in K$;

(2) $x \in K,-x \in K$ implies $x=0$.

Lemma 6. Let $E$ be a Banach space and let $K \subset E$ be a cone in $E$. Assume that $\Omega_{1}$ and $\Omega_{2}$ are open subsets of $E$ with $0 \in$ $\Omega_{1}, \bar{\Omega}_{1} \subset \Omega_{2}$. Let $T: K \cap\left(\bar{\Omega}_{2} \backslash \Omega_{1}\right) \rightarrow K$ be a completely continuous operator. In addition, suppose that either

(H1) $\|T u\| \leq\|u\|, \forall u \in K \cap \partial \Omega_{1}$ and $\|T u\| \geq\|u\|, \forall u \in$ $K \cap \partial \Omega_{2}$ or
(H2) $\|T u\| \geq\|u\|, \forall u \in K \cap \partial \Omega_{1}$ and $\|T u\| \leq\|u\|, \quad \forall u \in$ $K \cap \partial \Omega_{2}$

holds. Then $T$ has a fixed point in $K \cap\left(\bar{\Omega}_{2} \backslash \Omega_{1}\right)$.

In the following, we present the Green function of fractional differential equation boundary value problem.

Lemma 7 (see [4]). Let $y \in C[0,1]$, then the boundary value problem

$$
\begin{gathered}
D_{0+}^{\alpha} u(t)+y(t)=0, \quad 0<t<1, \\
u(0)=0, \quad \beta u(\eta)=u(1)
\end{gathered}
$$

has a unique solution

$$
u(t)=\int_{0}^{1} G(t, s) y(s) d s,
$$

where

$$
G(t, s)=\left\{\begin{array}{c}
\left([t(1-s)]^{\alpha-1}-\beta t^{\alpha-1}(\eta-s)^{\alpha-1}\right. \\
\left.-(t-s)^{\alpha-1}\left(1-\beta \eta^{\alpha-1}\right)\right) \\
\times\left(\left(1-\beta \eta^{\alpha-1}\right) \Gamma(\alpha)\right)^{-1}, \\
0 \leq s \leq \min \{t, \eta\} \leq 1 ; \\
\frac{[t(1-s)]^{\alpha-1}-(t-s)^{\alpha-1}\left(1-\beta \eta^{\alpha-1}\right)}{\left(1-\beta \eta^{\alpha-1}\right) \Gamma(\alpha)}, \\
0<\eta \leq s \leq t \leq 1 ; \\
\frac{[t(1-s)]^{\alpha-1}-\beta t^{\alpha-1}(\eta-s)^{\alpha-1}}{\left(1-\beta \eta^{\alpha-1}\right) \Gamma(\alpha)}, \\
0 \leq t \leq s \leq \eta<1 ; \\
\frac{[t(1-s)]^{\alpha-1}}{\left(1-\beta \eta^{\alpha-1}\right) \Gamma(\alpha)}, \\
0 \leq \max \{t, \eta\} \leq s \leq 1 .
\end{array}\right.
$$

Lemma 8. The function $G(t, s)$ defined by (11) satisfies $G(t, s)>0$ for $t, s \in(0,1)$.

Proof. (1) For $0 \leq s \leq \min \{t, \eta\} \leq 1$,

$G(t, s)$

$$
=\frac{[t(1-s)]^{\alpha-1}-\beta t^{\alpha-1}(\eta-s)^{\alpha-1}-(t-s)^{\alpha-1}\left(1-\beta \eta^{\alpha-1}\right)}{\left(1-\beta \eta^{\alpha-1}\right) \Gamma(\alpha)} .
$$

Let $F(t, s, \eta)=[t(1-s)]^{\alpha-1}-\beta t^{\alpha-1}(\eta-s)^{\alpha-1}-(t-s)^{\alpha-1}(1-$ $\left.\beta \eta^{\alpha-1}\right)$. There is

$$
\begin{aligned}
& \frac{\partial F(t, s, \eta)}{\partial \eta} \\
& =-\beta t^{\alpha-1}(\alpha-1)(\eta-s)^{\alpha-2}+(\alpha-1)(t-s)^{\alpha-1} \beta \eta^{\alpha-2} \\
& =\beta(\alpha-1)\left[(t-s)^{\alpha-1} \eta^{\alpha-2}-t^{\alpha-1}(\eta-s)^{\alpha-2}\right] \\
& \leq \beta(\alpha-1)\left[t^{\alpha-1} \eta^{\alpha-2}-t^{\alpha-1}(\eta-s)^{\alpha-2}\right] \\
& =\beta(\alpha-1) t^{\alpha-1}\left[\eta^{\alpha-2}-(\eta-s)^{\alpha-2}\right] .
\end{aligned}
$$


With $0<\beta<1,1<\alpha \leq 2$, there is $\partial F / \partial \eta<0$. Hence, function $F(t, s, \eta)$ is monotonically decreasing in $\eta$. With $0<$ $\eta<1, F(t, s, \eta)>F(t, s, \eta)_{\min }=F(t, s, 1)$. Let $\eta=1$, there is

$$
\begin{aligned}
F( & t, s, 1) \\
& =[t(1-s)]^{\alpha-1}-\beta t^{\alpha-1}(1-s)^{\alpha-1}-(t-s)^{\alpha-1}(1-\beta) \\
& =(1-\beta)\left\{[t(1-s)]^{\alpha-1}-(t-s)^{\alpha-1}\right\} \\
& =(1-\beta)\left[(t-t s)^{\alpha-1}-(t-s)^{\alpha-1}\right] .
\end{aligned}
$$

With $0<s \leq t<1,1<\alpha \leq 2$, we have $(t-t s)^{\alpha-1}-(t-s)^{\alpha-1} \geq$ 0 . With $1-\beta>0$, there is $F(t, s, 1) \geq 0$, then $F(t, s, \eta)>$ $F(t, s, 1) \geq 0$, there is $G(t, s)>0$.

(2) For $0<\eta \leq s \leq t<1$,

$$
G(t, s)=\frac{[t(1-s)]^{\alpha-1}-(t-s)^{\alpha-1}\left(1-\beta \eta^{\alpha-1}\right)}{\left(1-\beta \eta^{\alpha-1}\right) \Gamma(\alpha)} .
$$

Let $F(t, s, \eta)=[t(1-s)]^{\alpha-1}-(t-s)^{\alpha-1}\left(1-\beta \eta^{\alpha-1}\right)$; it is obvious that function $F(t, s, \eta)$ is monotonically increasing in $\eta$, when $\eta=0$,

$$
\begin{aligned}
F(t, s, \eta)_{\min } & =F(t, s, 0) \\
& =[t(1-s)]^{\alpha-1}-(t-s)^{\alpha-1} \\
& =(t-t s)^{\alpha-1}-(t-s)^{\alpha-1} .
\end{aligned}
$$

With $0 \leq s \leq t<1,0<\alpha-1 \leq 1$, there is $(t-t s)^{\alpha-1} \geq$ $(t-s)^{\alpha-1}$; we have $F(t, s, \eta)>F(t, s, 0) \geq 0$, then $G(t, s)>0$.

(3) For $0<t \leq s \leq \eta<1$,

$$
G(t, s)=\frac{[t(1-s)]^{\alpha-1}-\beta t^{\alpha-1}(\eta-s)^{\alpha-1}}{\left(1-\beta \eta^{\alpha-1}\right) \Gamma(\alpha)} .
$$

Let $F(t, s, \eta)=[t(1-s)]^{\alpha-1}-\beta t^{\alpha-1}(\eta-s)^{\alpha-1}$, then $F(t, s, \eta)$ is monotonically decreasing in $\eta$,

$$
\begin{aligned}
F(t, s, \eta)_{\min } & =F(t, s, 1) \\
& =[t(1-s)]^{\alpha-1}-\beta t^{\alpha-1}(1-s)^{\alpha-1} \\
& =(1-\beta)[t(1-s)]^{\alpha-1} .
\end{aligned}
$$

With $0<\beta<1,0<t \leq s<1$, there is $F(t, s, \eta)>0$, then $F(t, s, \eta)>F(t, s, 1) \geq 0$, hence $G(t, s)>0$.

(4) For $0 \leq \max \{t, \eta\} \leq s \leq 1$,

$$
G(t, s)=\frac{[t(1-s)]^{\alpha-1}}{\left(1-\beta \eta^{\alpha-1}\right) \Gamma(\alpha)}>0 .
$$

Therefore, $G(t, s)>0$ for $t, s \in(0,1)$. The proof is complete.

Lemma 9. $G(t, s) \leq G(s, s)$ for $t, s \in[0,1]$.
Proof. To get $G(t, s) \leq G(s, s)$, firstly, we prove the following inequality:

$$
\frac{t^{a}-s^{a}}{(t-s)^{a}} \leq \frac{1-s^{a}}{(1-s)^{a}}
$$

where $a=\alpha-1,0 \leq s<t \leq 1$. Let $f(t)=\left(t^{a}-s^{a}\right) /(t-s)^{a}$, then

$$
\begin{aligned}
f^{\prime}(t) & =\frac{a t^{a-1}(t-s)^{a}-a\left(t^{a}-s^{a}\right)(t-s)^{a-1}}{(t-s)^{2 a}} \\
& =\frac{a(t-s)^{a-1}}{(t-s)^{2 a}} \cdot\left[t^{a-1}(t-s)-\left(t^{a}-s^{a}\right)\right] \\
& =\frac{a(t-s)^{a-1}}{(t-s)^{2 a}} \cdot s\left(s^{a-1}-t^{a-1}\right) .
\end{aligned}
$$

With $1<\alpha \leq 2$, there is $a-1 \leq 0$; with $s<t$, there is $s^{a-1}>$ $t^{a-1}$; then $f^{\prime}(t)>0$. It means that $f(t)$ is monotonically increasing in $t$, then $f(t) \leq f(1)$; we have

$$
\frac{t^{a}-s^{a}}{(t-s)^{a}} \leq \frac{1-s^{a}}{(1-s)^{a}} .
$$

Here we prove $G(t, s) \leq G(s, s)$.

(1) For $0 \leq s \leq \min \{t, \eta\} \leq 1$,

$G(t, s)$

$$
\begin{gathered}
=\frac{[t(1-s)]^{\alpha-1}-\beta t^{\alpha-1}(\eta-s)^{\alpha-1}-(t-s)^{\alpha-1}\left(1-\beta \eta^{\alpha-1}\right)}{\left(1-\beta \eta^{\alpha-1}\right) \Gamma(\alpha)}, \\
G(s, s)=\frac{[s(1-s)]^{\alpha-1}-\beta s^{\alpha-1}(\eta-s)^{\alpha-1}}{\left(1-\beta \eta^{\alpha-1}\right) \Gamma(\alpha)} .
\end{gathered}
$$

With $\left(1-\beta \eta^{\alpha-1}\right) \Gamma(\alpha)>0, G(t, s) \leq G(s, s)$ is equivalent to

$$
\frac{t^{a}-s^{a}}{(t-s)^{a}}\left[(1-s)^{a}-\beta(\eta-s)^{a}\right] \leq 1-\beta \eta^{a}
$$

or

$\left(t^{a}-s^{a}\right)\left[(1-s)^{a}-\beta(\eta-s)^{a}\right] \leq\left(1-\beta \eta^{a}\right)(t-s)^{a}$.

(a) For $s=t, \quad\left(t^{a}-s^{a}\right)\left[(1-s)^{a}-\beta(\eta-s)^{a}\right]=\left(1-\beta \eta^{a}\right)(t-$ $s)^{a}=0$;

(b) for $s=t=\eta=1,\left(t^{a}-s^{a}\right)\left[(1-s)^{a}-\beta(\eta-s)^{a}\right]=$ $\left(1-\beta \eta^{a}\right)(t-s)^{a}=0$

(c) for $s=\eta \leq t$,

$$
\begin{gathered}
\left(t^{a}-s^{a}\right)\left[(1-s)^{a}-\beta(\eta-s)^{a}\right]=\left(t^{a}-\eta^{a}\right)\left(1-\eta^{a}\right), \\
\left(1-\beta \eta^{a}\right)(t-s)^{a}=\left(1-\beta \eta^{a}\right)(t-\eta)^{a},
\end{gathered}
$$

if $t=\eta,\left(t^{a}-s^{a}\right)\left[(1-s)^{a}-\beta(\eta-s)^{a}\right]=(1-$ $\left.\beta \eta^{a}\right)(t-s)^{a}=0$; if $t>\eta$, from (22), we have $\left(t^{a}-\eta^{a}\right) /$ $(t-\eta)^{a} \leq\left(1-\eta^{a}\right) /(1-\eta)^{a} \leq\left(1-\beta \eta^{a}\right) /(1-\eta)^{a}$; 
(d) for $0 \leq s<t \leq 1,0 \leq s<\eta \leq 1$, if $(1-s)^{a}-\beta(\eta-s)^{a}<$ 0 ; it is obvious that $\left(t^{a}-s^{a}\right)\left[(1-s)^{a}-\beta(\eta-s)^{a}\right] \leq$ $\left(1-\beta \eta^{a}\right)(t-s)^{a}$. If $(1-s)^{a}-\beta(\eta-s)^{a}>0$, by $(22)$, there is $\left(\eta^{a}-s^{a}\right) /(\eta-s)^{a} \leq\left(1-s^{a}\right) /(1-s)^{a}$; then

$$
(\eta-s)^{a} \geq \frac{1-s^{a}}{(1-s)^{a}}\left(\eta^{a}-s^{a}\right) .
$$

\section{Consider}

$$
\begin{aligned}
& \frac{t^{a}-s^{a}}{(t-s)^{a}}\left[(1-s)^{a}-\beta(\eta-s)^{a}\right] \\
& \quad \leq \frac{1-s^{a}}{(1-s)^{a}}\left[(1-s)^{a}-\beta(\eta-s)^{a}\right] \\
& \quad=1-s^{a}-\beta \frac{1-s^{a}}{(1-s)^{a}}(\eta-s)^{a} \\
& \quad \leq 1-s^{a}-\beta \frac{1-s^{a}}{(1-s)^{a}} \cdot \frac{(1-s)^{a}}{1-s^{a}} \cdot\left(\eta^{a}-s^{a}\right) \\
& \quad=1-\beta \eta^{a}-(1-\beta) s^{a} \\
& \quad \leq 1-\beta \eta^{a} .
\end{aligned}
$$

It means that $G(t, s) \leq G(s, s)$ holds.

(2) For $0<\eta \leq s \leq t \leq 1$,

$$
\begin{gathered}
G(t, s)=\frac{[t(1-s)]^{\alpha-1}-(t-s)^{\alpha-1}\left(1-\beta \eta^{\alpha-1}\right)}{\left(1-\beta \eta^{\alpha-1}\right) \Gamma(\alpha)}, \\
G(s, s)=\frac{[s(1-s)]^{\alpha-1}}{\left(1-\beta \eta^{\alpha-1}\right) \Gamma(\alpha)} .
\end{gathered}
$$

With $\left(1-\beta \eta^{\alpha-1}\right) \Gamma(\alpha)>0, G(t, s) \leq G(s, s)$ is equivalent to

$$
[t(1-s)]^{a}-(t-s)^{a}\left(1-\beta \eta^{a}\right) \leq[s(1-s)]^{a}
$$

or

$$
\left(t^{a}-s^{a}\right)(1-s)^{a} \leq(t-s)^{a}\left(1-\beta \eta^{a}\right) .
$$

From (22), we have $\left(\left(t^{a}-s^{a}\right) /(t-s)^{a}\right)(1-s)^{a} \leq 1-s^{a}$; with $\eta \leq s, 0<\beta<1$, we have $1-s^{a} \leq 1-\eta^{a} \leq 1-\beta \eta^{a}$; then $\left(\left(t^{a}-s^{a}\right) /(t-s)^{a}\right)(1-s)^{a} \leq 1-\beta \eta^{a}$; with $s \leq t$, there is

$$
\left(t^{a}-s^{a}\right)(1-s)^{a} \leq(t-s)^{a}\left(1-\beta \eta^{a}\right) .
$$

It means that $G(t, s) \leq G(s, s)$ holds.

(3) For $0 \leq t \leq s \leq \eta<1$,

$$
\begin{aligned}
& G(t, s)=\frac{[t(1-s)]^{\alpha-1}-\beta t^{\alpha-1}(\eta-s)^{\alpha-1}}{\left(1-\beta \eta^{\alpha-1}\right) \Gamma(\alpha)}, \\
& G(s, s)=\frac{[s(1-s)]^{\alpha-1}-\beta s^{\alpha-1}(\eta-s)^{\alpha-1}}{\left(1-\beta \eta^{\alpha-1}\right) \Gamma(\alpha)} .
\end{aligned}
$$

With $\left(1-\beta \eta^{\alpha-1}\right) \Gamma(\alpha)>0, G(t, s) \leq G(s, s)$ is equivalent to

$$
[t(1-s)]^{a}-\beta t^{a}(\eta-s)^{a} \leq[s(1-s)]^{a}-\beta s^{a}(\eta-s)^{a}
$$

or

$$
\left(t^{a}-s^{a}\right)(1-s)^{a} \leq \beta\left(t^{a}-s^{a}\right)(\eta-s)^{a} .
$$

With $0<\beta<1, s \leq \eta$, there is $\beta(\eta-s)^{a} \leq(\eta-s)^{a}<(1-s)^{a}$; with $t \leq s$, there is

$$
\left(t^{a}-s^{a}\right)(1-s)^{a} \leq \beta\left(t^{a}-s^{a}\right)(\eta-s)^{a} .
$$

It means that $G(t, s) \leq G(s, s)$ holds.

(4) For $0<\max \{t, \eta\} \leq s \leq 1$,

$$
\begin{aligned}
& G(t, s)=\frac{[t(1-s)]^{\alpha-1}}{\left(1-\beta \eta^{\alpha-1}\right) \Gamma(\alpha)}, \\
& G(s, s)=\frac{[s(1-s)]^{\alpha-1}}{\left(1-\beta \eta^{\alpha-1}\right) \Gamma(\alpha)} .
\end{aligned}
$$

It is obvious that $G(t, s) \leq G(s, s)$. The proof is complete.

Lemma 10. $G(t, s) \geq q(k) G(s, s)$ for $t, s \in[1 / k, 1-(1 / k)]$, where $k>2$ is an integer and

$$
\begin{aligned}
q(k)=\min \left\{\frac{(1-\beta)\left[(1-(1 / k))^{2(\alpha-1)}-(1-(2 / k))^{\alpha-1}\right]}{(1 / 4)^{\alpha-1}},\right. \\
\\
\frac{(1-\beta)(1 / k)^{2(\alpha-1)}}{(1 / 4)^{\alpha-1}}, \\
\\
\left.\frac{(k-1)^{\alpha-1} / k^{2(\alpha-1)}}{(1 / 4)^{\alpha-1}}\right\} .
\end{aligned}
$$

Proof. From (11), we have

$$
G(s, s)= \begin{cases}\frac{[s(1-s)]^{\alpha-1}-\beta s^{\alpha-1}(\eta-s)^{\alpha-1}}{\left(1-\beta \eta^{\alpha-1}\right) \Gamma(\alpha)}, & s \leq \eta, \\ \frac{[s(1-s)]^{\alpha-1}}{\left(1-\beta \eta^{\alpha-1}\right) \Gamma(\alpha)}, & s>\eta .\end{cases}
$$

It is obvious that $[s(1-s)]^{\alpha-1} /\left(1-\beta \eta^{\alpha-1}\right) \Gamma(\alpha) \geq$ $\left([s(1-s)]^{\alpha-1}-\beta s^{\alpha-1}(\eta-s)^{\alpha-1}\right) /\left(1-\beta \eta^{\alpha-1}\right) \Gamma(\alpha)$, for $t, s \in[1 / k, 1-(1 / k)]$,

$$
\begin{aligned}
\max G(s, s) & =\left.\frac{[s(1-s)]^{\alpha-1}}{\left(1-\beta \eta^{\alpha-1}\right) \Gamma(\alpha)}\right|_{s=1 / 2} \\
& =\frac{(1 / 4)^{\alpha-1}}{\left(1-\beta \eta^{\alpha-1}\right) \Gamma(\alpha)} .
\end{aligned}
$$

(1) For $1 / k \leq s \leq \min \{t, \eta\} \leq 1-(1 / k)$, let

$$
\begin{aligned}
F(t, s, \eta)= & {[t(1-s)]^{\alpha-1}-\beta t^{\alpha-1}(\eta-s)^{\alpha-1} } \\
& -(t-s)^{\alpha-1}\left(1-\beta \eta^{\alpha-1}\right) .
\end{aligned}
$$


By Lemma 8 , for $\eta=1$,

$$
\begin{aligned}
& \bar{F}(t, s)= F(t, s, \eta)_{\min }=F(t, s, 1) \\
&= {[t(1-s)]^{\alpha-1}-\beta t^{\alpha-1}(1-s)^{\alpha-1} } \\
&-(t-s)^{\alpha-1}(1-\beta) \\
&=(1-\beta)\left\{[t(1-s)]^{\alpha-1}-(t-s)^{\alpha-1}\right\} \\
&=(1-\beta)\left[(t-t s)^{\alpha-1}-(t-s)^{\alpha-1}\right], \\
& \frac{\partial \bar{F}}{\partial s}=(1-\beta)(\alpha-1)\left[(t-s)^{\alpha-2}-t^{\alpha-1}(1-s)^{\alpha-2}\right] .
\end{aligned}
$$

With $1<\alpha \leq 2,0 \leq s \leq t \leq 1$, then $(t-s)^{\alpha-2}-t^{\alpha-1}(1-$ $s)^{\alpha-2}>0$; with $0<\beta<1, \partial \bar{F} / \partial s>0$. It means that $\bar{F}(t, s)$ is monotonically increasing in $s$. Similarly,

$$
\frac{\partial \bar{F}}{\partial t}=(1-\beta)(\alpha-1)\left[(t-t s)^{\alpha-2}(1-s)-(t-s)^{\alpha-2}\right]<0 .
$$

It means that $\bar{F}(t, s)$ is monotonically decreasing in $t$. Therefore

$$
\begin{aligned}
\bar{F}(t, s) & \geq \min \bar{F}(t, s)=\left.\bar{F}(t, s)\right|_{t=1-(1 / k), s=1 / k} \\
& =(1-\beta)\left[\left(1-\frac{1}{k}\right)^{2(\alpha-1)}-\left(1-\frac{2}{k}\right)^{\alpha-1}\right],
\end{aligned}
$$

$\min G(t, s)$

$$
\begin{gathered}
=\frac{(1-\beta)\left[\left((1 / k)-\left(1 / k^{2}\right)\right)^{\alpha-1}-(1-(2 / k))^{\alpha-1}\right]}{\left(1-\beta \eta^{\alpha-1}\right) \Gamma(\alpha)}, \\
\frac{\min G(t, s)}{\max G(s, s)}=\frac{(1-\beta)\left[(1-(1 / k))^{2(\alpha-1)}-(1-(2 / k))^{\alpha-1}\right]}{(1 / 4)^{\alpha-1}} .
\end{gathered}
$$

(2) For $1 / k<\eta \leq s \leq t \leq 1-(1 / k)$, let

$$
F(t, s, \eta)=[t(1-s)]^{\alpha-1}-(t-s)^{\alpha-1}\left(1-\beta \eta^{\alpha-1}\right) .
$$

By Lemma 8 , for $\eta=0$,

$$
\begin{aligned}
\bar{F}(t, s) & =F(t, s, \eta)_{\min }=F(t, s, 0) \\
& =[t(1-s)]^{\alpha-1}-(t-s)^{\alpha-1} \\
& =(t-t s)^{\alpha-1}-(t-s)^{\alpha-1}, \\
\frac{\partial \bar{F}}{\partial s}=(\alpha-1) & {\left[(t-s)^{\alpha-2}-t^{\alpha-1}(1-s)^{\alpha-2}\right]>0 . }
\end{aligned}
$$

It means that $\bar{F}(t, s)$ is monotonically increasing in $s$

$$
\frac{\partial \bar{F}}{\partial t}=(\alpha-1)\left[(1-s)^{\alpha-1} t^{\alpha-2}-(t-s)^{\alpha-2}\right]<0 .
$$

It means that $\bar{F}(t, s)$ is monotonically decreasing in $t$

$$
\begin{aligned}
\bar{F}(t, s) & \geq \min \bar{F}(t, s)=\left.\bar{F}(t, s)\right|_{t=1-(1 / k), s=(1 / k)} \\
& =\left(1-\frac{1}{k}\right)^{2(\alpha-1)}-\left(1-\frac{2}{k}\right)^{\alpha-1}, \\
\min G(t, s) & =\frac{(1-\beta)\left[(1-(1 / k))^{2(\alpha-1)}-(1-(2 / k))^{\alpha-1}\right]}{\left(1-\beta \eta^{\alpha-1}\right) \Gamma(\alpha)}, \\
\frac{\min G(t, s)}{\max G(s, s)} & =\frac{(1-\beta)\left[(1-(1 / k))^{2(\alpha-1)}-(1-(2 / k))^{\alpha-1}\right]}{(1 / 4)^{\alpha-1}} .
\end{aligned}
$$

(3) For $1 / k \leq t \leq s \leq \eta<1-(1 / k)$, let

$$
F(t, s, \eta)=[t(1-s)]^{\alpha-1}-\beta t^{\alpha-1}(\eta-s)^{\alpha-1} .
$$

By Lemma 8 , for $\eta=1$,

$$
\begin{aligned}
\bar{F}(t, s) & =F(t, s, \eta)_{\min }=F(t, s, 1) \\
& =[t(1-s)]^{\alpha-1}-\beta t^{\alpha-1}(1-s)^{\alpha-1} \\
& =(1-\beta)[t(1-s)]^{\alpha-1}, \\
\frac{\partial \bar{F}}{\partial s}= & (\alpha-1) t^{\alpha-1}(\beta-1)(1-s)^{\alpha-2}<0 ;
\end{aligned}
$$

then $\bar{F}(t, s)$ is monotonically decreasing in $s$. Consider

$$
\frac{\partial \bar{F}}{\partial t}=(\alpha-1)(1-\beta) t^{\alpha-2}(1-s)^{\alpha-1}>0 ;
$$

then $\bar{F}(t, s)$ is monotonically increasing in $t$. Therefore,

$$
\begin{aligned}
\bar{F}(t, s) \geq & \min \bar{F}(t, s)=\left.\bar{F}(t, s)\right|_{t=1 / k, s=1-(1 / k)}=\frac{1-\beta}{k^{2(\alpha-1)}}, \\
\min G(t, s) & =\frac{(1-\beta)\left(1 / k^{2(\alpha-1)}\right)}{\left(1-\beta \eta^{\alpha-1}\right) \Gamma(\alpha)}, \\
\frac{\min G(t, s)}{\max G(s, s)} & =\frac{(1-\beta)(1 / k)^{2(\alpha-1)}}{(1 / 4)^{\alpha-1}} .
\end{aligned}
$$

(4) For $1 / k \leq \max \{t, \eta\} \leq s \leq 1-(1 / k)$, let

$$
F(t, s)=[t(1-s)]^{\alpha-1} .
$$

It is obvious that $F(t, s)$ is monotonically increasing in $t$ and monotonically decreasing in $s$; then

$$
\begin{aligned}
F(t, s) & \geq \min F(t, s)=\left.F(t, s)\right|_{t=1 / k, s=1-(1 / k)} \\
& =\left(\frac{1}{k}-\frac{1}{k^{2}}\right)^{\alpha-1} ;
\end{aligned}
$$

we have

$$
\min G(t, s)=\frac{\left((1 / k)-\left(1 / k^{2}\right)\right)^{\alpha-1}}{\left(1-\beta \eta^{\alpha-1}\right) \Gamma(\alpha)}
$$


there is

$$
\frac{\min G(t, s)}{\max G(s, s)}=\frac{(k-1)^{\alpha-1} / k^{2(\alpha-1)}}{(1 / 4)^{\alpha-1}}
$$

Let

$$
\begin{aligned}
q(k)=\min \left\{\frac{(1-\beta)\left[(1-(1 / k))^{2(\alpha-1)}-(1-(2 / k))^{\alpha-1}\right]}{(1 / 4)^{\alpha-1}},\right. \\
\\
\frac{(1-\beta)(1 / k)^{2(\alpha-1)}}{(1 / 4)^{\alpha-1}}, \\
\left.\frac{(k-1)^{\alpha-1} / k^{2(\alpha-1)}}{(1 / 4)^{\alpha-1}}\right\} .
\end{aligned}
$$

Therefore, $G(t, s) / G(s, s) \geq \min G(t, s) / \max G(s, s)=q(k) \neq$ 0 .

The proof is complete.

We consider the Banach space $X=C[0,1]$ equipped with standard norm $\|u\|=\max _{0 \leq t \leq 1}|u(t)|, u \in X$. We define a cone $P$ by

$$
\begin{array}{r}
P=\{u \in X: u(t) \geq 0, t \in[0,1], \\
\left.\min _{t \in[1 / k, 1-1 / k]} u(t) \geq q(k)\|u\|\right\} .
\end{array}
$$

Define an integral operator $T: P \rightarrow X$ by

$$
\begin{array}{r}
(T u)(t)=\lambda \int_{0}^{1} G(t, s) a(s) f(s, u(s)) d s, \\
0 \leq t \leq 1, \quad u \in P .
\end{array}
$$

Lemma 11. It holds the following.

(1) $T: P \rightarrow P$ is completely continuous.

(2) $u(t)$ is a positive solution of the fractional boundary value problem (4) if and only if $u(t)$ is a fixed point of the operator $T$ in cone $P$.

Proof. For $u \in P, t \in[1 / k, 1-(1 / k)]$, by Lemma $10, G(t, s) \geq$ $q(k) G(s, s)$; for $t, s \in[1 / k, 1-(1 / k)]$,

$$
\begin{aligned}
T u(t) & =\lambda \int_{0}^{1} G(t, s) a(s) f(s, u(s)) d s \\
& \geq \lambda q(k) \int_{0}^{1} G(s, s) a(s) f(s, u(s)) d s \\
& =\lambda q(k) \max _{1 / k \leq t \leq 1-1 / k} \int_{0}^{1} G(t, s) a(s) f(s, u(s)) d s \\
& =q(k)\|T u(t)\| .
\end{aligned}
$$

It is obvious that $\min _{1 / k \leq t \leq 1-1 / k}(T u)(t) \geq q(k)\|T u\|, T P \subseteq P$. Thus $T P \subset P$. In addition, standard arguments show that $T$ is completely continuous.
(2) It is obvious that $u(t)$ is the positive solution of BVP (4) if and only if

$$
u(t)=\lambda \int_{0}^{1} G(t, s) a(s) f(s, u(s)) d s, \quad 0 \leq t \leq 1
$$

It can be proved by the definition of integral operator $T$.

\section{Main Results}

We denote some important constants as follows:

$$
\begin{gathered}
A=\int_{0}^{1} G(s, s) a(s) q(k) d s, \\
B=\int_{0}^{1} G(s, s) a(s) d s, \\
F_{0}=\lim _{u \rightarrow 0^{+}} \sup \max _{0 \leq t \leq 1} \frac{f(t, u(t))}{u(t)}, \\
f_{0}=\lim _{u \rightarrow+\infty} \inf \min _{0 \leq t \leq 1} \frac{f(t, u(t))}{u(t)}, \\
F_{\infty}=\lim _{u \rightarrow 0^{+}} \sup \max _{0 \leq t \leq 1} \frac{f(t, u(t))}{u(t)}, \\
f_{\infty}=\lim _{u \rightarrow+\infty} \inf \min _{0 \leq t \leq 1} \frac{f(t, u(t))}{u(t)} .
\end{gathered}
$$

Here we assume that $1 / A f_{\infty}=0$ if $f_{\infty} \rightarrow \infty, 1 / B F_{0}=\infty$ if $F_{0} \rightarrow 0,1 / A f_{0}=0$ if $f_{0} \rightarrow \infty$, and $1 / B F_{\infty}=\infty$ if $F_{\infty} \rightarrow 0$.

Theorem 12. Suppose that $A f_{\infty}>B F_{0}$, then for each $\lambda \in$ $\left(1 / A f_{\infty}, 1 / B F_{0}\right), B V P(4)$ has at least one positive solution.

Proof. We choose $\varepsilon>0$ sufficiently small such that $\left(F_{0}+\right.$ $\varepsilon) \lambda B \leq 1$. By the definition of $F_{0}$, we can see that there exists $l_{1}>0$, such that $f(t, u(t)) \leq\left(F_{0}+\varepsilon\right) u(t)$ for $0 \leq u \leq l_{1}$. For $u \in P$ with $\|u\|=l_{1}$, we have

$$
\begin{aligned}
\|T u(t)\| & =\lambda \int_{0}^{1} G(s, s) a(s) f(s, u(s)) d s \\
& \leq \lambda \int_{0}^{1} G(s, s) a(s)\left(F_{0}+\varepsilon\right) u(s) d s \\
& \leq \lambda\left(F_{0}+\varepsilon\right)\|u\| \int_{0}^{1} G(s, s) a(s) d s \\
& =\lambda B\left(F_{0}+\varepsilon\right)\|u\| \\
& \leq\|u\| .
\end{aligned}
$$

Then we have $\|T u\| \leq\|u\|$. Thus if we let $\Omega_{1}=\{u \in X:\|u\|<$ $\left.l_{1}\right\}$, then $\|T u\| \leq\|u\|$ for $u \in P \cap \partial \Omega_{1}$. We choose $\delta>0$ and $c \in(0,1 / 4)$ such that $\lambda\left(f_{\infty}-\delta\right) A \geq 1$. There exists $l_{2}>l_{1}>0$, 
such that $f(t, u) \geq\left(f_{\infty}-\delta\right) u$ for $u>l_{2}$. Therefore, for each $u \in P$ with $\|u\|=l_{2}$, we have

$$
\begin{aligned}
\|T u(t)\| & =\lambda \int_{0}^{1} G(s, s) a(s) f(s, u(s)) d s \\
& \geq \lambda \int_{c}^{1} G(s, s) a(s)\left(f_{\infty}-\delta\right) u(s) d s \\
& \geq \lambda\left(f_{\infty}-\delta\right)\|u\| \int_{c}^{1} G(s, s) a(s) q(k) d s \\
& \geq \lambda\left(f_{\infty}-\delta\right) A \cdot\|u\| \\
& \geq\|u\| .
\end{aligned}
$$

Thus if we let $\Omega_{2}=\left\{u \in E:\|u\|<l_{2}\right\}$, then $\bar{\Omega}_{1} \subset$ $\Omega_{2}$ and $\|T u\| \geq\|u\|$ for $u \in P \cap \partial \Omega_{2}$. Condition (H1) of Krasnoesel'skii's fixed point theorem is satisfied. So there exists a fixed point of $T$ in $P$. This completes the proof.

Theorem 13. Suppose that $A f_{0}>B F_{\infty}$, then for each $\lambda \epsilon$ $\left(1 / A f_{0}, 1 / B F_{\infty}\right), B V P(4)$ has at least one positive solution.

Proof. Choose $\varepsilon>0$ sufficiently small such that $\left(f_{0}-\varepsilon\right) \lambda A \geq$ 1. From the definition of $f_{0}$, we see that there exists $l_{1}>0$, such that $f(t, u) \geq\left(f_{0}-\varepsilon\right) u$ for $0<u \leq l_{1}$. If $u \in P$ with $\|u\|=l_{1}$, we have

$$
\begin{aligned}
\|T u(t)\| & =\lambda \int_{0}^{1} G(s, s) a(s) f(s, u(s)) d s \\
& \geq \lambda\left(f_{0}-\varepsilon\right)\|u\| A \\
& \geq\|u\| .
\end{aligned}
$$

Let $\Omega_{1}=\left\{u \in X:\|u\|<l_{1}\right\}$; then we have $\|T u\| \geq\|u\|$ for $u \in P \cap \partial \Omega_{1}$. Choose $\delta>0, c \in(0,1 / 4)$; then we have $\lambda\left(F_{\infty}+\delta\right) A \leq 1$. There exists $l_{2}>l_{1}>0$, such that $f(t, u) \leq$ $\left(F_{\infty}+\delta\right) u$ for $u>l_{2}$. For each $u \in P$ with $\|u\|=l_{2}$, we have

$$
\begin{aligned}
\|T u(t)\| & =\lambda \int_{0}^{1} G(s, s) a(s) f(s, u(s)) d s \\
& \leq \lambda \int_{c}^{1} G(s, s) a(s)\left(F_{\infty}+\delta\right) u(s) d s \\
& \leq \lambda\left(F_{\infty}+\delta\right)\|u\| \int_{c}^{1} G(s, s) a(s) q(k) d s \\
& \leq \lambda\left(F_{\infty}+\delta\right) A \cdot\|u\| \\
& \leq\|u\| .
\end{aligned}
$$

Let $\Omega_{2}=\left\{u \in E:\|u\|<l_{2}\right\}$; then $\bar{\Omega}_{1} \subset \Omega_{2}$, and we have $\|T u\| \leq\|u\|$ for $u \in P \cap \partial \Omega_{2}$. Condition (H2) of Krasnoesel'skii's fixed-point theorem is satisfied. So there exists a fixed point of $T$ in $P$. This completes the proof.

\section{An Example}

Example 1. Consider the following three-point fractional boundary value problem:

$$
\begin{aligned}
D_{0+}^{3 / 2} u(t)+\lambda f(t, u(t)) & =0, \quad 0<t<1, \\
u(0)=0, \quad u(1) & =\frac{1}{2} u\left(\frac{1}{3}\right),
\end{aligned}
$$

where $f(t, u(t))=\left((t+1)\left(2014 u^{3}+u\right) /\left(u^{2}+1\right)\right)+u(\sin u+$ 1), $a(t)=1$.

By calculations,

$$
G(s, s)=\left\{\begin{array}{c}
\frac{[s(1-s)]^{\alpha-1}-\beta s^{\alpha-1}(\eta-s)^{\alpha-1}}{\left(1-\beta \eta^{\alpha-1}\right) \Gamma(\alpha)}, \\
0 \leq s=t \leq \eta \leq 1, \\
\frac{[s(1-s)]^{\alpha-1}}{\left(1-\beta \eta^{\alpha-1}\right) \Gamma(\alpha)}, \\
0 \leq \eta \leq t=s \leq 1,
\end{array}\right.
$$

$$
\begin{aligned}
\int_{0}^{1} G(s, s) d s= & \int_{0}^{\eta} \frac{[s(1-s)]^{\alpha-1}-\beta s^{\alpha-1}(\eta-s)^{\alpha-1}}{\left(1-\beta \eta^{\alpha-1}\right) \Gamma(\alpha)} d s \\
& +\int_{\eta}^{1} \frac{[s(1-s)]^{\alpha-1}}{\left(1-\beta \eta^{\alpha-1}\right) \Gamma(\alpha)} d s \\
= & \int_{0}^{1} \frac{[s(1-s)]^{\alpha-1}}{\left(1-\beta \eta^{\alpha-1}\right) \Gamma(\alpha)} d s \\
& -\int_{0}^{\eta} \frac{\beta s^{\alpha-1}(\eta-s)^{\alpha-1}}{\left(1-\beta \eta^{\alpha-1}\right) \Gamma(\alpha)} d s \\
= & \quad-(1 / 2)(1 / 3)^{1 / 2} \Gamma(3 / 2) \\
& \times\left\{\int_{0}^{1}[s(1-s)]^{1 / 2} d s\right. \\
& \left.-\int_{0}^{1 / 3} \frac{1}{2} s^{1 / 2}(\eta-s)^{1 / 2} d s\right\}
\end{aligned}
$$$$
=0.58832 \text {. }
$$

Let $k=10$, then

$$
\begin{aligned}
q(k) \min \left\{\frac{(1-\beta)\left[(1-(1 / k))^{2(\alpha-1)}-(1-(2 / k))^{\alpha-1}\right]}{(1 / 4)^{\alpha-1}},\right. \\
\frac{(1-\beta)(1 / k)^{2(\alpha-1)}}{(1 / 4)^{\alpha-1}}, \\
\left.\frac{(k-1)^{\alpha-1} / k^{2(\alpha-1)}}{(1 / 4)^{\alpha-1}}\right\} .
\end{aligned}
$$$$
=0.00557 \text {, }
$$ 


$$
\begin{aligned}
f_{\infty} & =\lim _{u \rightarrow+\infty} \inf \min _{0 \leq t \leq 1}\left[\frac{(t+1)\left(2014 u^{2}+1\right)}{u^{2}+1}+\sin u+1\right] \\
& =2014, \\
F_{0} & =\lim _{u \rightarrow 0^{+}} \sup \max _{0 \leq t \leq 1}\left[\frac{(t+1)\left(2014 u^{2}+1\right)}{u^{2}+1}+\sin u+1\right] \\
& =4 .
\end{aligned}
$$

Consider

$$
\begin{gathered}
A=\int_{0}^{1} G(s, s) a(s) q(k) d s=0.00328 \\
B=\int_{0}^{1} G(s, s) a(s) d s=0.58832 .
\end{gathered}
$$

The condition $A f_{\infty}>B F_{0}$ is obtained. From Theorem 12, we see that if $\lambda \in(0.15138,0.42494)$, the problem (67) has a positive solution.

\section{Conflict of Interests}

The authors declare that there is no conflict of interests regarding the publication of this paper.

\section{Acknowledgments}

The authors are grateful to the reviewers for the valuable suggestions. This paper was supported by the Fundamental Research Funds for the Central Universities (no. 2652012141) and Beijing Higher Education Young Elite Teacher Project.

\section{References}

[1] Z. Bai and H. Lü, "Positive solutions for boundary value problem of nonlinear fractional differential equation," Journal of Mathematical Analysis and Applications, vol. 311, no. 2, pp. 495-505, 2005.

[2] R. P. Agarwal, D. O’Regan, and S. Staněk, "Positive solutions for Dirichlet problems of singular nonlinear fractional differential equations," Journal of Mathematical Analysis and Applications, vol. 371, no. 1, pp. 57-68, 2010.

[3] D. Delbosco and L. Rodino, "Existence and uniqueness for a nonlinear fractional differential equation," Journal of Mathematical Analysis and Applications, vol. 204, no. 2, pp. 609-625, 1996.

[4] Z. Bai, "On positive solutions of a nonlocal fractional boundary value problem," Nonlinear Analysis: Theory, Methods \& Applications, vol. 72, no. 2, pp. 916-924, 2010.

[5] R. P. Agarwal, M. Benchohra, and S. Hamani, "A survey on existence results for boundary value problems of nonlinear fractional differential equations and inclusions," Acta Applicandae Mathematicae, vol. 109, no. 3, pp. 973-1033, 2010.

[6] M. El-Shahed, "Positive solutions for boundary value problem of nonlinear fractional differential equation," Abstract and Applied Analysis, vol. 2007, Article ID 10368, 8 pages, 2007.
[7] X. Xu, D. Jiang, and C. Yuan, "Multiple positive solutions for the boundary value problem of a nonlinear fractional differential equation," Nonlinear Analysis: Theory, Methods \& Applications, vol. 71, no. 10, pp. 4676-4688, 2009.

[8] B. Ahmad, "Existence of solutions for irregular boundary value problems of nonlinear fractional differential equations," Applied Mathematics Letters, vol. 23, no. 4, pp. 390-394, 2010.

[9] S. Liang and J. Zhang, "Positive solutions for boundary value problems of nonlinear fractional differential equation," Nonlinear Analysis: Theory, Methods \& Applications, vol. 71, no. 11, pp. 5545-5550, 2009.

[10] O. K. Jaradat, A. Al-Omari, and S. Momani, "Existence of the mild solution for fractional semilinear initial value problems," Nonlinear Analysis: Theory, Methods \& Applications, vol. 69, no. 9, pp. 3153-3159, 2008. 


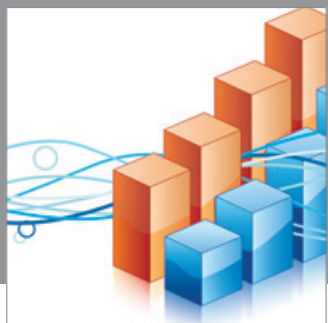

Advances in

Operations Research

mansans

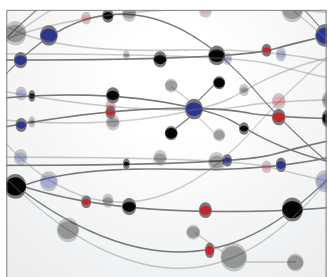

The Scientific World Journal
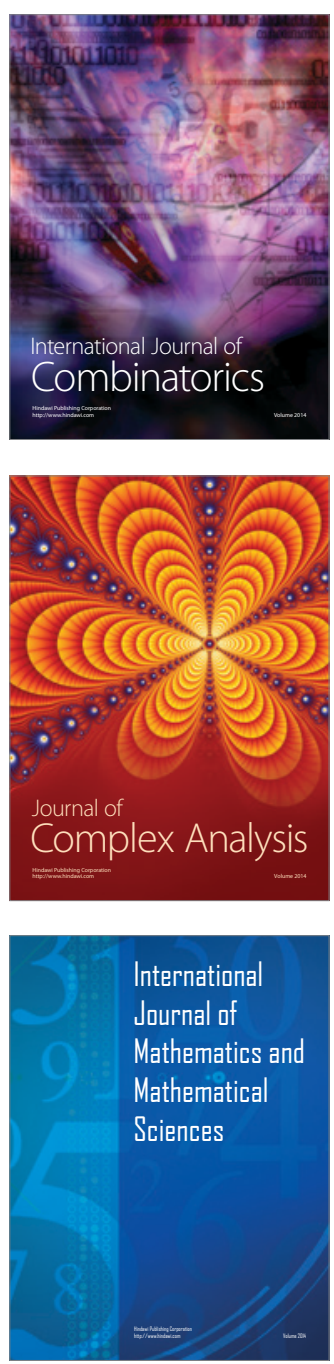
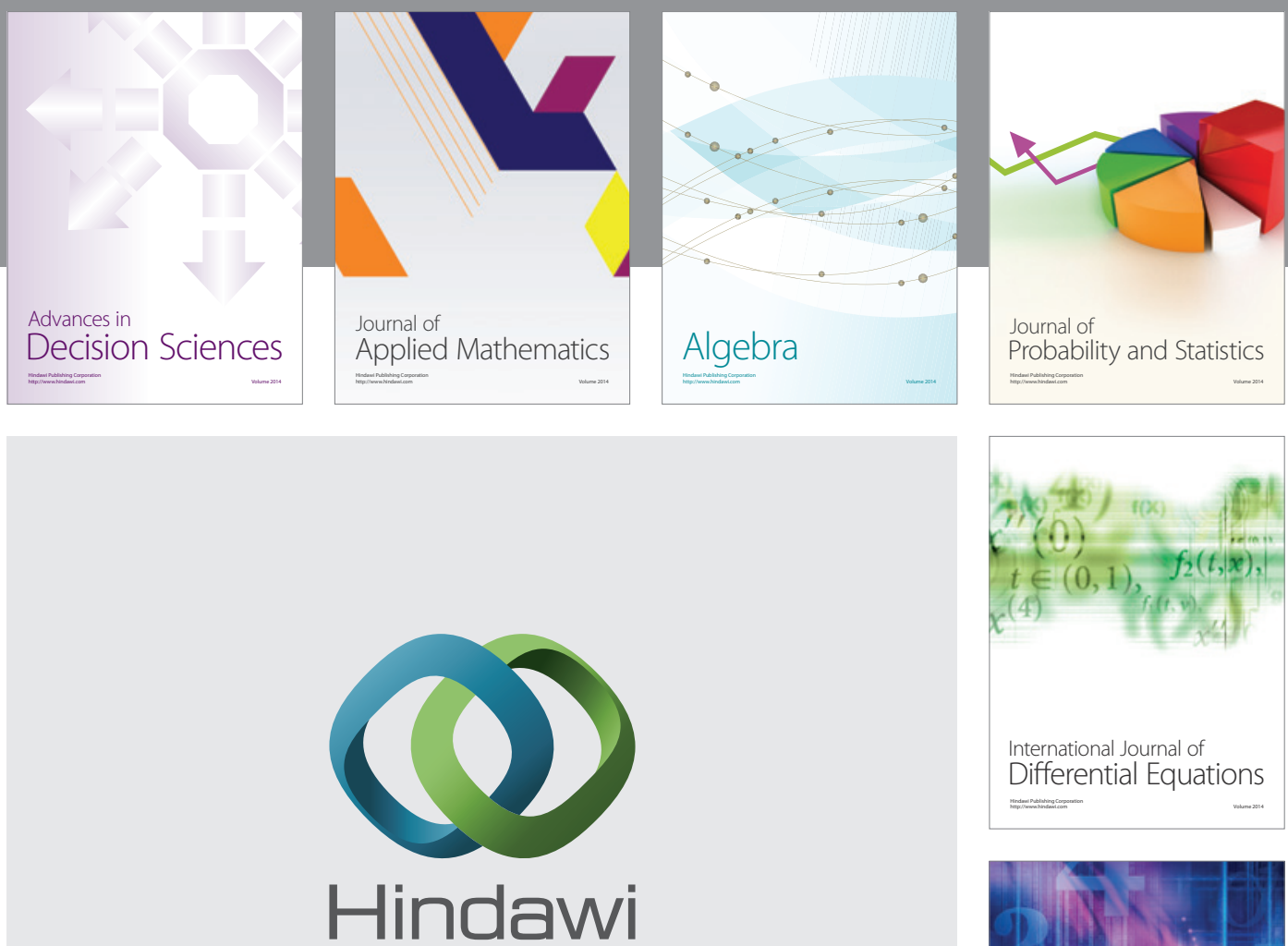

Submit your manuscripts at http://www.hindawi.com
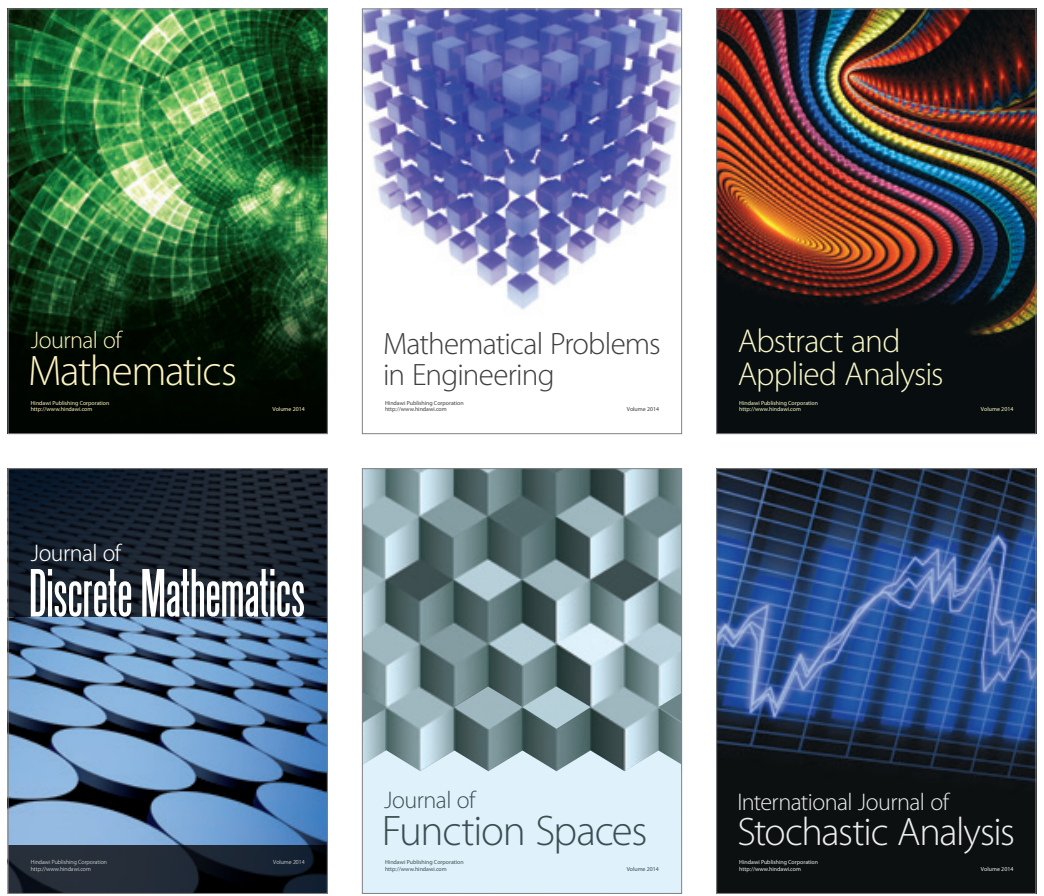

Journal of

Function Spaces

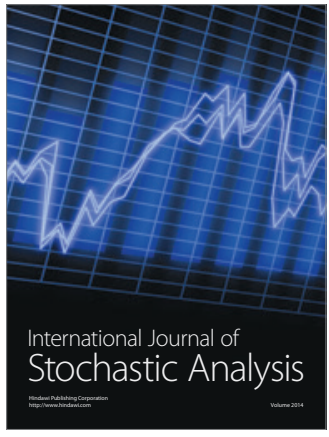

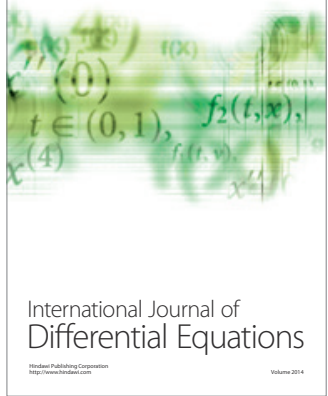
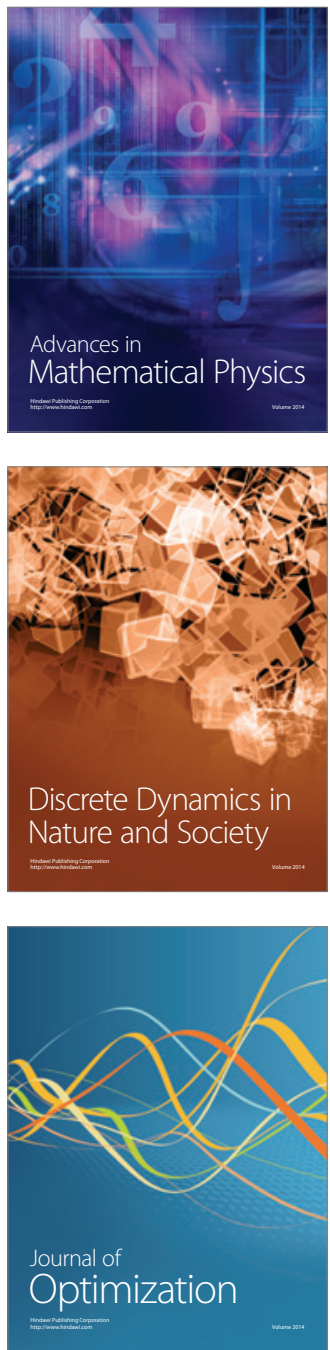